\title{
An Overview of Drug Master Files
}

\section{Albert Yehaskel*}

EAS Consulting Group, LLC, USA

*Corresponding author: Albert Yehaskel, Independent Consultant, EAS Consulting Group, LLC, 1700 Diagonal Road, Suite 750, Alexandria, VA 22314, USA, Tel: 571-447-5508; E-mail: ascanlin@easconsultinggroup.com

Received Date: Dec 26, 2017; Accepted Date: Jan 10, 2018; Published Date: Jan 18, 2017

Copyright: @ 2018 Yehaskel A. This is an open-access article distributed under the terms of the Creative Commons Attribution License, which permits unrestricted use, distribution and reproduction in any medium, provided the original author and source are credited.

\section{Introduction}

A Drug Master File (DMF) is an elective regulatory submission and is submitted at the discretion of the DMF holder to assist their clients. In the absence of relevant information in the CMC section of an application, the US Food and Drug Administration requires a Drug Master File submission of a drug substance, drug product, and/or container closure submission to allow FDA to review information such as confidential details about facilities, processes, components, or articles used in the manufacturing, processing, packaging, and storing of one or more APIs and/or human drugs. These submissions support a third party's application without revealing the information to the third party and maintains the confidentiality of proprietary information (e.g., a synthetic or manufacturing procedure) for the holder, allowing review of information by reviewers at FDA to support applications submitted by one or more applications. The information contained in a DMF may be used to support an Investigational New Drug Application (IND), a New Drug Application (NDA/BLA), an Abbreviated New Drug Application (ANDA), another DMF, an Export Application, and any amendments and supplements to any of these applications.

A Drug Master File is submitted to the Center for Drug Evaluation and Research as it is different from a Biologics Master File which gets submitted to the Center for Biological Evaluation and Research.

\section{DMF Types}

There are five types of DMFs, the most common being a Type II DMF followed by a Type III DMF. It should be noted, however, that only four DMFs (types II-V) are still actively submitted as the Type I DMF has been phased out.

Type II: Drug substances (Active Pharmaceutical Ingredients), substance intermediates and materials used in their preparation, or a drug product. A Type II DMF, the most common form, can also cover dosage form drugs manufactured under contract for another company which would file an ANDA.

Type III: Packaging materials, from bottles and caps to PVC resin used in their manufacture must be covered in a DMF or other FDA document such as an NDA.

Type IV: Excipient, colorant, flavor, essence or material DMF. Excipients are chemically inactive substances such as starches or cellulose used to bind drug powder together so that it can be pressed into a tablet. Other examples include flavorings in children's drugs, alcohol in liquids, etc.

Type V: This is basically FDA accepted reference information that is not included in the other types of DFMFs.
Finally, there is also a Type I DMF which, as noted earlier, is no longer accepted by the FDA. However, older ones remain on file.

It should be noted that the manufacturer of any materials (e.g, API, DP, container/closure components, etc.) can choose to submit the information necessary for review directly to their customers for inclusion in the IND, NDA, ANDA, and BLA, supplements or amendments to these applications. This can be accomplished in whole or in part. If only a part is submitted, it is considered the "open part" of a DMF that is shared, while the proprietary part is the "closed part" of the DMF.

\section{Regulatory Basis of Drug Master Files}

The submission of a DMF is not required by law or FDA regulation but is only submitted at the discretion of the holder. Once submitted, a DMF is not approved or disapproved; the contents of a DMF are reviewed only in connection with the review of an application such as an IND, NDA, ANDA and supplements/amendments. Additionally, information will also get reviewed when an IND sponsor, an applicant for an NDA, ANDA or another DMF holder incorporates material in the DMF by reference.

If FDA reviewers find deficiencies in the information provided in a DMF, a letter describing the deficiencies is sent to the DMF holder. FDA will also notify the person who relies on the information in the deficient DMF that additional information is needed in the supporting DMF.

FDA discusses requirements for filing a DMF under the Code of Federal Regulations called 21 CFR 314.420 or Applications for FDA Approval to Market a New Drug.

\section{Contents of a DMF Submission}

There are certain requirements for each DMF submission, such as a transmittal letter, administrative information about the submission, and the specific information to be included in the DMF all of which must be written in English. DMFs from overseas firms that originate in a language other than English must be accurately translated with a certification of translation included.

Aside from the User Fee Form, no other forms should be filled out or submitted along with a DMF submission. Letters of Authorization (LOAs) submitted with initial DMF submission must contain the DMF number.

Each page of each copy of the DMF should be dated and consecutively numbered and any updates should include an updated table of contents.

The transmittal letter for an original DMF should cover the following: 
- Identification of submission: Original, the type of DMF, and its subject;

- Identification of the applications, if known, that the DMF is intended to support, including the name and address of each sponsor, applicant, or holder, and all relevant document numbers;

- Signature of the holder, authorized representative or agent;

- Name and title of the signer.

The administrative information required is as follows: The names and addresses of the following must be provided:

- DMF holder;

- Corporate headquarters;

- Manufacturing/processing facility;

- Contact for FDA correspondence; this is the contact person and a telephone number, fax number and email address should be provided;

- Agent(s), if any;

- The specific responsibilities of each person;

- A statement of commitment;

- A signed statement by the holder certifying that the DMF is current and that the DMF holder will comply with the statements made in it.

The FDA regulations regarding DMFs states: "Any addition, change, or deletion of information in a drug master file is required to be submitted in two copies and to describe by name, reference number, volume, and page number the information affected in the drug master file".

The FDA ensures that DMFs are current. If a company has not submitted an annual report for three years, the agency sends an "Overdue Notification Letters" to DMF holders. The holder has 90 days in which to respond and submit its annual report. If they fail to respond, their DMF may be closed.

A note when filing new DMFs, FDA requires and provides a preassigned application number. While a pre-assigned number will be required for a new DMF, those converting paper DMF submissions to electronic (eDMF) may use the originally provided DMF number as the submission information is the same, only the vehicle for submission has changed.

(Please note that in cases where the originally assigned DMF number is fewer than six digits, zeros must be added to the beginning of the number to conform to the 6-digit format.)

Information on how to obtain a pre-assigned number can be found at FDA's website: "Requesting a Pre-assigned Application number":

http://www.fda.gov/Drugs/DevelopmentApprovalProcess/FormsSubmission Requirements/ElectronicSubmissions/ucm114027.htm

(It should be noted that a request for a pre-assigned DMF number for a Type V DMF should include documentation that the request for a Type V was cleared.)

Many firms choose to have DMF information reviewed by a regulatory consultant who specializes in pharmaceuticals and the DMF process prior to submission to verify all DMF parts are included and any all technical information is thorough and complete.

\section{The Mechanism of a Drug Master File}

A DMF goes through two stages of being assessed prior to it being available for review of its [technical] content. First, FDA assesses whether all parts of the DMF are included and in the correct order. Once FDA determines that the eDMF is acceptable, it will then undergo an administrative review as discussed above. If the DMF is not acceptable from an electronic technical perspective, the holder will be informed. The holder must then satisfactorily respond to any deficiencies for the DMF to proceed to an administrative review which will be conducted by the DMF staff in the Office of Pharmaceutical Quality (OPQ). If the DMF passes the administrative review and is found to be acceptable, OPQ sends an Acknowledgement Letter at which point the DMF is available for review of the technical content. However, if the DMF is not acceptable from an administrative point of view, OPQ sends an Administrative Filing Issues (AFI) letter. The holder must respond adequately for the DMF to be available for review of the technical content. The time frame for this could be from 2-3 weeks.

\section{Electronic Submissions}

Starting May 5, 2018, any new submissions to the existing DMF must be assembled and submitted in eCTD format. If a current DMF is in paper format with the FDA, the same submission does not need to be resubmitted in eCTD format. This is specified in FDA's eCTD guidance: "Guidance for Industry: Providing Regulatory Submissions in Electronic Format-Certain Human Pharmaceutical Product Applications and Related Submissions Using the eCTD Specifications" (May 2015). Please refer to the following website:

http://www.fda.gov/downloads/Drugs/

GuidanceComplianceRegulatoryInformation/Guidances/UCM333969.pdf

It should be noted that DMF's that are not submitted in eCTD format will now be rejected by the Agency. DMF holders whose DMFs are currently in paper format will not be required to resubmit their entire DMFs in eCTD format after May 5, 2018, only amendments and updates.

It should also be noted that electronic submissions that are $10 \mathrm{~GB}$ or smaller in size must be submitted through the Electronic Submission Gateway (ESG). The majority of DMF submissions generally fall into this category. Towards that end, it is recommended that submitters obtain an ESG account or discuss their submission options with consulting firms which offer this service. Submissions that are over 10 GB may be submitted on physical media (such as compact disc). Please refer to FDA's eCTD web page for additional information:

http://www.fda.gov/Drugs/DevelopmentApprovalProcess/ FormsSubmissionRequirements/ElectronicSubmissions/ucm153574.htm

\section{US Agents}

The US FDA requires that all foreign firms conducting business in the FDA regulatory sphere do so with the use of a U.S. Agent, who acts as an intermediary between FDA and the foreign firm. US Agent information must be included in all DMF submissions to FDA and any updates of US Agent contact information should be submitted as well. Should FDA reviewers have questions regarding the DMF, they will contact the US Agent with those questions in addition to the submitter and provide a timeline in which those responses must be provided. 
DMF submissions are technical reports to FDA concerning a number of variables in the drug application, all of which relate to the safety and efficacy of pharmaceutical products marketed in the US. Close attention should be paid to not only the content but the format in which the documentation is provided to FDA. It is likely that FDA will have follow-on questions of the submitter, but a competent US Agent as well as competent review of the materials by a qualified pharmaceutical consultant with expertise in the drug submission process will make the process go more smoothly.

One final caveat-DMF's must be updated when new information is available. The new information could be a new plasticizer for a container/closure system, new route of synthesis, an improvement to a dosage form, or any other change that has the potential for compromising the safety of the pharmaceutical. It is incumbent upon DMF holders to communicate any changes in advance of the change to their clients to allow them time to make their own plans and changes. This is critical! It is also important that any deficiency letters sent by the FDA to the DMF holder be communicated to their clients who can be impacted by the deficiency letter. The DMF holder needs to respond to the deficiencies in the most expeditious manner to prevent FDA holding back approvals of submissions. This too is very critical. A consultant could be invaluable in demystifying these types of issues. 